\title{
Signature of wide-spread clumping in B supergiant winds
}

\author{
R. K. Prinja ${ }^{1}$ and D. L. Massa ${ }^{2}$ \\ 1 Department of Physics \& Astronomy, UCL, Gower Street, London WC1E 6BT, UK \\ e-mail: rkp@star.ucl.ac.uk \\ 2 Space Telescope Science Institute, 3700 San Marino Drive, Baltimore, MD 21218, USA \\ e-mail: massa@stsci.edu
}

Received 22 June 2010 / Accepted 15 July 2010

\begin{abstract}
Aims. We seek to establish additional observational signatures of the effects of clumping in OB star winds. The action of clumping on strategic wind-formed spectral lines is tested to steer the development of models for clumped winds and thus improve the reliability of mass-loss determinations for massive stars.

Methods. The SirV $\lambda \lambda 1400$ resonance line doublets of B0 to B5 supergiants are analysed using empirical line-synthesis models. The focus is on decoding information on wind clumping from measurements of ratios of the radial optical depths $\left(\tau_{\text {rad }}(w)\right)$ of the red and blue components of the Sirv doublet. We exploit in particular the fact that the two doublet components are decoupled and formed independently for targets with relatively low wind terminal velocities.

Results. Line-synthesis analyses reveal that the mean ratio of $\tau_{\text {rad }}(w)$ of the blue to red Sirv components are rarely close to the canonical value of $\sim 2$ (expected from atomic constants), and spread instead over a range of values between $\sim 1$ and 2 . These results are interpreted in terms of a photosphere that is partially obscured by optically thick structures in the outflowing gas.

Conclusions. The spectroscopic signatures established in this study demonstrate the wide-spread existence of wind clumping in B supergiants. The additional information in unsaturated doublet profiles provides a means to quantify the porosity of the winds.
\end{abstract}

Key words. stars: early-type - stars: mass-loss - ultraviolet: stars

\section{Introduction}

There are current serious uncertainties in the mass-loss and thus energy feedback processes of massive OB stars. Over the past decade evidence has accumulated from a broad collection of results to strongly challenge the current model of hot star mass-loss via stellar winds. Of particular impact on mass-loss determinations is the presence of (small-scale) clumping and (large-scale) structure, resulting in porous winds. The characteristics of the X-ray emission (e.g. Cohen 2008), excess flux at IR and mm wavelengths (Runacres \& Blomme 1996), discordance between FUV (sensitive to density, $\rho$ ) and $\mathrm{H} \alpha$ (sensitive to $\rho^{2}$ ) measurements of mass-loss rates (e.g. Massa et al. 2003; Bouret et al. 2005; Fullerton et al. 2006; Puls et al. 2006) collectively suggest the winds are highly clumped, with a consequent reduction in actual mass-loss rates. Given that some of these studies question mass-loss rates of massive stars at the order-ofmagnitude level, there is the highest urgency to robustly investigate this discordance since it has potentially far reaching consequences for the evolution and fate of massive stars (which is largely dependent on mass-loss) and galactic chemical evolution (where mass-loss drives feedback into the interstellar medium).

Furthermore, recent theoretical predictions of the effects of structure in porous winds (Oskinova et al. 2007; Sundqvist et al. 2010), ionization by shock produced XUV line radiation (Waldron \& Cassinelli 2010) and X-rays (Krticka et al. 2009) have amplified the need to verify via additional methods the compelling FUV studies cited above, which were based on the PV $\lambda \lambda 1118,1128$ doublet.

\section{The Si IV $\lambda \lambda 1400$ resonance line doublet ratios}

In this letter we report on additional, untapped information on the nature of wind clumping that can be extracted from an analysis of the doublet ratios of SiIV $\lambda \lambda 1393.76,1402.77$ in B supergiants. The AGN community have previously recognised the potential of ratios of doublet components as a signature of clumping (e.g. Ganguly et al. 1999). The reason is that for a source obscured by a uniform distribution of gas, the ratio of optical depths determined from the observed absorption lines would equal the ratio of the oscillator strengths of the two doublet components. However, in the case of a source that is partially obscured by totally opaque clumps, the ratios of observed optical depth will be 1 , since they will depend only on the fraction of the source which is uncovered.

We exploit this diagnostic here (in an extension to Massa et al. 2008): in a homogeneous massive star wind, the ratio of the optical depths of the widely spaced SiIV $\lambda \lambda 1400$ doublet would be the usual ratio of the oscillator strengths of the two components (i.e. 2.01). However, in an OB star wind composed of very optically thick clumps embedded in a transparent medium, the radial optical depth of an absorption line depends only on the geometric covering factor of the clumps. Therefore in this case reducing the optical depths of the clumps by a factor of 2 (as implied in the SiIV doublets) makes minimal difference in the absorption. Thus in this clumped case the ratio of doublet optical depths measured from the absorption lines would lie between $\sim 1$ and 2. 
Table 1. SiIV SEI model profile fitting parameters for the core sample.

\begin{tabular}{llllllllcc}
\hline \hline Star & Sp. type & $T_{\text {eff }}(\mathrm{kK})$ & $v_{\infty}$ & $\beta$ & $v_{\text {turb }}$ & $\tau_{\text {rad }}(w)($ blue $/$ red $)$ & $\begin{array}{c}\left\langle\dot{M} q_{i}\right\rangle(\mathrm{blue}) \\
\left(10^{-9} M_{\odot} \mathrm{yr}^{-1}\right)\end{array}$ & $\begin{array}{l}\left\langle\dot{M} q_{i}\right\rangle(\mathrm{red}) \\
\left(10^{-9} M_{\odot} \mathrm{yr}^{-1}\right)\end{array}$ \\
\hline HD 13866 & B2 Ib-II:p & 18.8 & 860 & 0.7 & 0.03 & 1.45 & 0.52 & 0.70 \\
HD 14134 & B3 Ia & 16.5 & 495 & 0.5 & 0.10 & 1.37 & 0.83 & 1.39 \\
HD 14143 & B2 Ia & 18.0 & 525 & 3.5 & 0.25 & 1.80 & 7.46 & 8.36 \\
HD 36371 & B4 Ib & 16.5 & 465 & 0.8 & 0.17 & 1.10 & 0.38 & 0.46 \\
HD 42087 & B2.5 Ib & 18.0 & 630 & 1.2 & 0.11 & 1.04 & 0.66 & 1.24 \\
HD 43384 & B3 Iab & 16.5 & 630 & 0.9 & 0.20 & 1.19 & 1.11 & 2.82 \\
HD 47240 & B1 Ib & 20.5 & 955 & 1.0 & 0.07 & 1.04 & 1.36 & 2.99 \\
HD 51309 & B3 Ib & 16.5 & 730 & 0.5 & 0.15 & 1.55 & 0.94 & 1.81 \\
HD 53138 & B3 Ia & 16.5 & 515 & 1.1 & 0.28 & 1.22 & 1.97 & 2.28 \\
HD 58350 & B5 Ia & 15.0 & 380 & 0.8 & 0.25 & 2.04 & 0.90 & 1.25 \\
HD 58510 & B1 Ib-II & 20.5 & 915 & 0.9 & 0.19 & 1.79 & 1.37 & 1.62 \\
HD 77581 & B0.5 Iae & 26.0 & 655 & 1.0 & 0.22 & 1.80 & 1.19 & 1.72 \\
HD 79186 & B5 Ia & 15.0 & 435 & 0.8 & 0.23 & 1.73 & 0.86 & 1.09 \\
HD 93827 & B1 Ib & 20.5 & 590 & 0.5 & 0.16 & 1.64 & 0.10 & 0.15 \\
HD 111934 & B1.5 Ib & 18.5 & 935 & 3.1 & 0.15 & 1.46 & 4.62 & 6.35 \\
HD 111973 & B3 Ia & 16.5 & 520 & 0.5 & 0.25 & 1.43 & 0.87 & 1.31 \\
HD 111990 & B2 Ib & 18.0 & 715 & 3.3 & 0.12 & 1.97 & 2.14 & 2.50 \\
HD 119608 & B1 Ib & 20.5 & 925 & 0.6 & 0.25 & 1.14 & 2.05 & 3.69 \\
HD 148379 & B2 Iap & 18.0 & 560 & 3.2 & 0.17 & 1.32 & 3.78 & 9.86 \\
HD 152236 & B0.5 Ia & 26.0 & 470 & 2.0 & 0.31 & 1.12 & 1.42 & 2.03 \\
HD 152667 & B0 Ia & 27.5 & 800 & 2.5 & 0.28 & 1.78 & 4.44 & 4.81 \\
HD 155985 & B0.7 Ib & 23.5 & 940 & 0.5 & 0.13 & 1.24 & 0.60 & 0.95 \\
HD 157246 & B1 Ib & 20.5 & 600 & 0.5 & 0.31 & 1.08 & 0.67 & 1.56 \\
HD 198478 & B2.5 Ia & 17.5 & 560 & 2.0 & 0.24 & 1.34 & 2.14 & 4.16 \\
HD 225094 & B3 Ia & 16.5 & 485 & 0.5 & 0.21 & 1.75 & 0.81 & 0.78 \\
\hline
\end{tabular}

Notes. $T_{\text {eff }}$ from Searle et al. (2008). The $\left\langle\dot{M} q_{i}\right\rangle$ values include a few cases where $\tau_{\text {rad }}(w)$ exceeds 5.0 in isolated velocity bins.

\section{The B supergiant sample}

The dataset of SirV resonance line doublets analysed in this study is derived from the B0 to B5 supergiants discussed by Prinja et al. (2005), using high resolution International Ultraviolet Explorer (IUE) satellite spectra. In particular our core sample of targets are B supergiants that (i) show well-developed but unsaturated SiIV absorption troughs; and (ii) have wind terminal velocities that are less than 0.5 of the SiIV doublet separation; i.e. stars with $v_{\infty} \lesssim 970 \mathrm{~km} \mathrm{~s}^{-1}$. This is an important selection criterion since it means that the two components of the doublet can be treated as radiatively decoupled and can be analysed separately i.e. each component as a singlet. The core sample of 25 stars fitting our primary criteria above are listed in Table 1.

To extract physical information from the line profiles we employed the "Sobolev with Exact Integration" (SEI) code of Lamers et al. (1987), but with the modifications described by Massa et al. (2003), which permit the radial optical depth $\left(\tau_{\text {rad }}(w)\right)$ to be treated as 21 discrete, independent and variable bins in velocity space. The bins are then adjusted to match the observed profile by a non-linear least squares procedure. The often complex photospheric spectrum in the region of SiIV $\lambda \lambda 1400$ was included in the SEI fitting by using the IUE spectra of $d$ warf B stars listed in Prinja et al. (2002). (The B dwarf spectra do not exhibit SiIV wind lines and were rotationally broadened to match the target B supergiants.)

By model fitting the blue and red Sirv components as singlets, we are then able to extract two independent sets of $\tau_{\text {rad }}(w)$, which can be inspected for signatures of wind clumping (as justified in Sect. 2). The SEI model fitting parameters, including $v_{\infty}$, velocity law index $(\beta)$ and turbulent velocity parameter $\left(v_{\text {turb }}\right)$ are also listed in Table 1, for the 25 B supergiants for which the Sirv doublet components can be considered as decoupled.

\section{The evidence for clumping}

Three representative examples of the Sirv blue and red components independently matched by SEI models are shown in Fig. 1, covering the spectral type range B0.7 to B4. The corresponding radial optical depths are also plotted for each case. The ratio of the blue-to-red optical depths, averaged between 0.2 to $0.9 v_{\infty}$, is shown for the full sample of 25 stars in Fig. 2 (filled circles; listed in Table 1). For reliability, we only consider optical depths in the interval $0.3 \leq \tau_{\text {rad }}(w) \leq 5.0$, to avoid weaker and saturated lines. We assign an error of $\pm 5 \%$ in the $\tau_{\text {rad }}(w)$ values adopted in each velocity bin. It is clear that in almost all cases the best fits for the decoupled doublet components yield optical depth ratios less than 2.01 (the expected value from atomic constants). The majority of ratios lie between $\sim 1.0$ and 1.5 , and the overall mean is $\sim 1.46$ (s.d. $\sim 0.31$ ). As argued in Sect. 2, blue-to-red Sirv doublet $\tau_{\text {rad }}(w)$ ratios significantly lower than $\sim 2$ imply that the B supergiant winds are porous and contain very optically thick clumps. Note in Fig. 2 that the ratios do not depend on the effective temperature of the relatively wide B0 to B5 spectral type range, and we therefore rule out any systematic effects that would cause all of the derived ratios to differ in the same manner. Our results clearly indicate that the weaker (red) component of the SiIV doublet is stronger than expected from smooth wind models.

An alternative measure of the departure from the smooth wind predictions is listed in Table 1, where the product of massloss rate $\times \mathrm{Si}^{3+}$ ion fraction $\left(\dot{M} q_{i}\right)$ is provided for each doublet component, as determined from the SEI fits (see formulation in Massa et al. 2003). (The values in Table 1 are integrated over $0.2 \leq v / v_{\infty} \leq 0.9$.) In almost all cases the ratios of the blue to red $\langle\dot{M} q\rangle$ measurements lie between 0.5 and 1.0. These results differ from the optical depth ratios shown in Fig. 2 because 
R. K. Prinja and D. L. Massa: Signature of wide-spread clumping in B supergiant winds
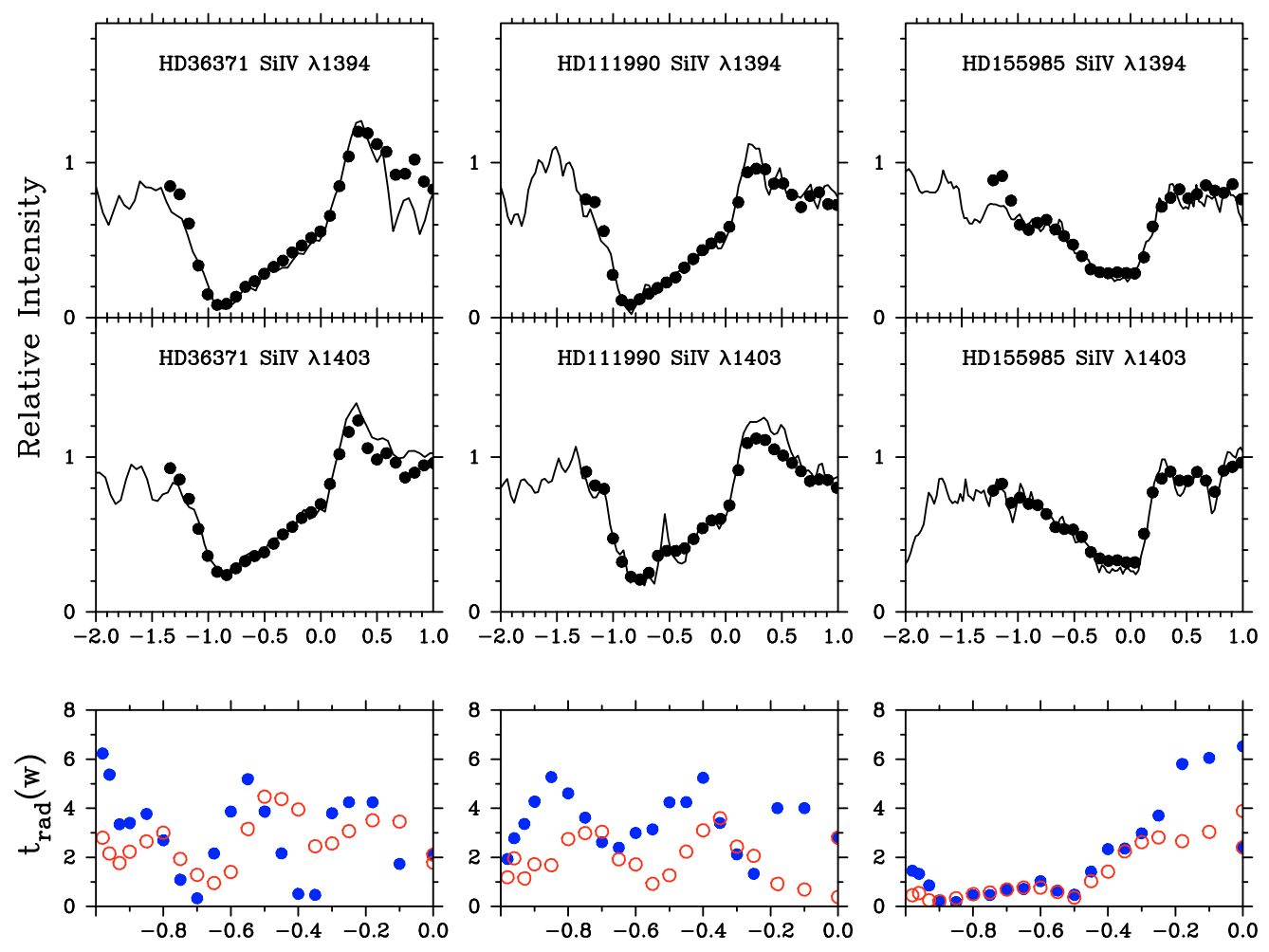

\section{Normalised velocity (w)}

Fig. 1. Examples of SEI line synthesis fits to SiIV $\lambda 1393.76$ (upper panels) and SiIV $\lambda 1402.77$ (middle panels) in our core B supergiant sample. The lower-most panels show the corresponding radial optical depths for the blue (filled circles) and red (open circles) doublet components.

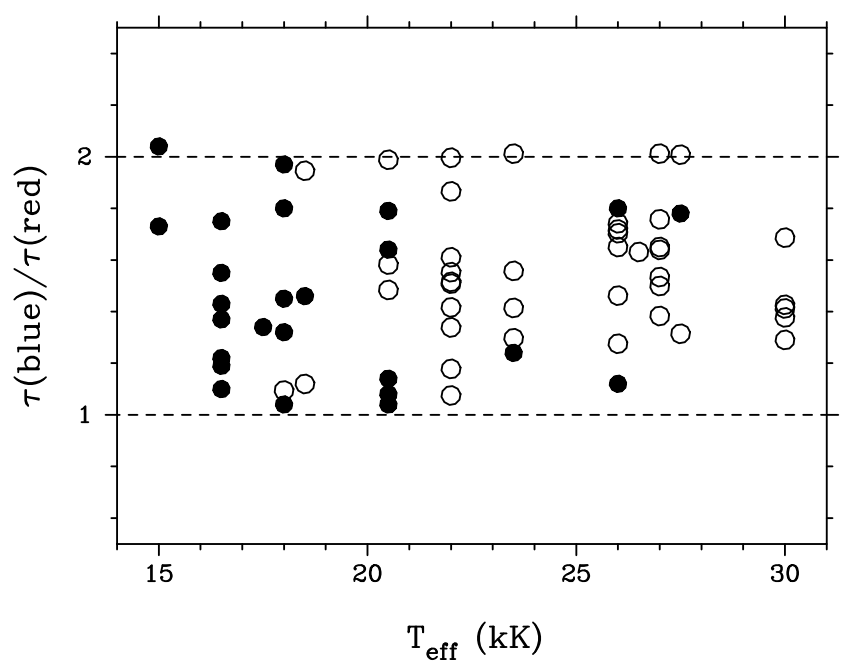

Fig. 2. Filled circles - the mean optical depth ratio between 0.2 and $0.9 v_{\infty}$ for Sirv $\lambda 1394 /$ SiIV $\lambda 1403$ versus effective temperature, for our core sample of 25 stars, where $v_{\infty}$ is less than half the doublet separation. Only optical depths in the range 0.3 to 5.0 are considered, to avoid poor quality and saturated cases, respectively. Open circles - plot of the derived oscillator strength ratios versus effective temperature for additional B supergiants (Sect. 4.1), where $v_{\infty}$ exceeds more than half the SirV doublet separation. In this case, the ratio of oscillator strengths was a free parameter in the fits.

$\dot{M} q=$ const $x^{2} w \frac{\mathrm{d} w}{\mathrm{~d} x} \tau(w) / \lambda f$ (where const contains stellar and atomic data which are the same for both components). This relation has two consequences. First, the ratios of the $\langle\dot{M} q\rangle$ scale as $(\lambda f)_{\text {blue }} /(\lambda f)_{\text {red }} \simeq 2$ times the ratio of the mean optical depths, which accounts for difference in range. Second, the determinations of the mean values in Table 1 are weighted differently, which accounts for the fact that mean $\tau_{\text {rad }}(w)$ ratio entry is not exactly 2 times $\langle\dot{M} q\rangle_{\text {blue }} /\langle\dot{M} q\rangle_{\text {red }}$.

\subsection{Additional data sample}

The evidence for widespread wind clumping in B supergiants presented here (Fig. 2) is based on the robust case of well separated, decoupled Sirv components, i.e. for stars that have $v_{\infty} \leq$ $970 \mathrm{~km} \mathrm{~s}^{-1}$. There are of course many additional B supergiants with available UV data that have $v_{\infty}$ in excess of $\sim 1000 \mathrm{~km} \mathrm{~s}^{-1}$. As the terminal velocity in these cases exceeds more than half the SiIV doublet separation, their doublet components cannot be matched independently as "singlets" (as above). We can nevertheless extract some complementary information from these higher $v_{\infty}$ stars.

From the original B supergiant IUE sample of Prinja et al. (2005), we selected an additional 41 B0 to B2 stars and fitted the (now coupled) SiIV doublets with the SEI models (see Massa et al. 2008). However, in these cases the ratio of the doublet oscillator strengths was allowed to be a free parameter in the least squares fits. The best fits to the sample of overlapping SiIV doublets yielded $f$-value ratios that also lie between $\sim 1$ and 2 in almost all cases. The results are also shown in Fig. 2 (open circles) as a function of $T_{\text {eff. }}$. For a fixed mass-loss rate, velocity law and ion fraction, the ratio of blue-to-red oscillator strengths is equivalent to the ratio of optical depths. Therefore the results shown in Fig. 2 are consistent with the original sample (Table 1 and Fig. 2 filled circles) of low $v_{\infty}$, decoupled doublets. Taken together, we 
conclude that the two datasets make a compelling case for widespread clumping and porosity in B supergiant winds.

\section{Discussion}

We have demonstrated that the SiIV $\lambda \lambda 1400$ doublets of B supergiants contain additional signatures of clumping in the stellar winds. Line synthesis models of 25 B0 to B5 stars for which the SiIV blue and red components can be treated as decoupled ("singlets"), yield blue-to-red optical depth ratios between 1 and 2, i.e. mostly well below the value $\sim 2$ expected from atomic constants and a smooth wind. These results may be interpreted as evidence for optically thick clumps in the wind, covering only a fraction of the stellar source. Additional results derived from B stars with overlapping SiIV doublets are consistent with this conclusion.

The optical depth ratios (Fig. 2) are not dependent on the effective temperature. The large scatter observed in the ratio for a given spectral type bin is also not correlated with the projected stellar rotation velocity or mass-loss rate. Aside from inclination effects connected to large-scale structures such as co-rotating interaction regions, the scatter in Fig. 2 may reflect transient and episodic events in the wind. It may be expected that for highly porous and extremely clumped cases, the temporal effect due to the time-dependent arrangement of clumps would be more pronounced. As an illustration, we examined the $I U E$ time-series dataset of HD 47240 (B1 Ib; Table 1) described by Prinja et al. (2002), to test for temporal variations in the mean blue-to-red optical depth ratios. Thirteen SiIV spectra (SWP48835 to 48953), spanning $\sim 16$ days, were matched by SEI models according to the methods for decoupled doublets outlined in Sect. 3. The mean blue-to-red $\tau_{\text {rad }}(w)$ ratio measured over 0.2 to $0.9 v_{\infty}$ varies considerably during the 16 day monitoring of HD 47240, with values between $\sim 1.2$ to 2.0 , i.e. essentially the full range of scatter seen in Fig. 2 at a given spectral type. Consequently, we suspect that much of the scatter seen in Fig. 2 results from timevariable covering factors. The spectroscopic changes modelled here in HD 47240 are empirically due to time-variable velocity dependent structure in the UV absorption line profiles. Several previous time-series studies of variations in the UV resonance lines of OB stars have interpreted this temporal behaviour as due to the evolution and velocity migration of large-scale structure in the winds, perhaps due to density inhomogeneities or velocity plateaus. The substantial UV line profile changes evident in OB stars are not diagnostics of the effects of small-scale mircoclumping. The inference here then may be that the variable blueto-red $\tau_{\text {rad }}(w)$ ratios derived from the UV resonance lines of an individual OB star reflect the porosity of a macro-clumped wind. We now plan additional studies to derive information about the spatial extent and temporal evolution of the obscuring structure, which may be decoded from the suspected wavelength dependence of observed changes in the mean $\tau_{\text {rad }}(w)$ ratios.

To a large degree the current debate concerning clumping in porous winds, and the consequent over-estimation of clumping independent mass-loss rates, is founded on observational and theoretical studies of O-type stars (see e.g. references in Sect. 1). The line doublets diagnostics that we have demonstrated here for B supergiants can to some extent also be tested on O-type stars. Since the terminal velocities of O-type stars are mostly higher than for B supergiants, only the method for overlapping

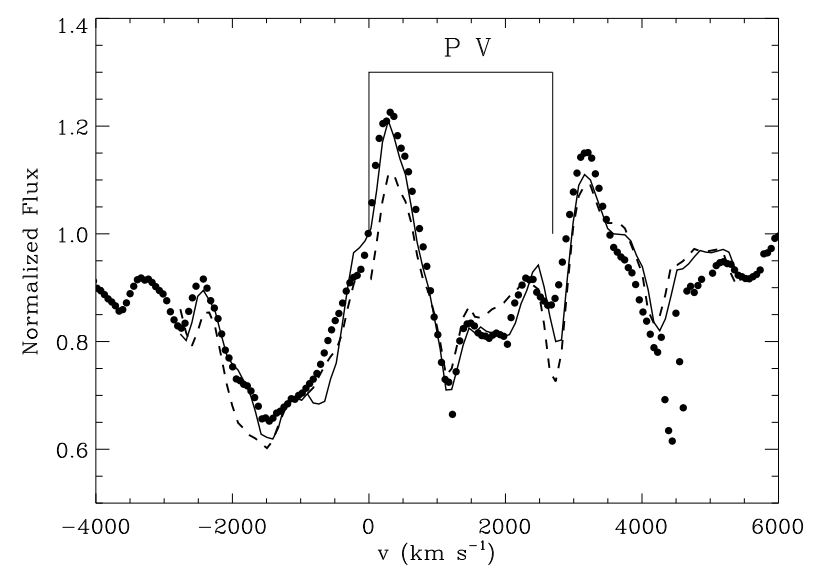

Fig. 3. Plot of the observed Pv $\lambda \lambda 1117,1128$ resonance line doublet in the O4 supergiant $\zeta$ Pup (dotted line). The dashed line fit is for the oscillator strength ratio fixed by the atomic constants $(=2.02)$. The solid line is the improved match obtained by allowing the $f$-ratio to be a free parameter ( $=1.84$ for the minimized residuals).

doublets employed in Sect. 4.1 is feasible for O stars, i.e. allowing the $f$-ratio of the doublet to vary from the value expected from atomic constants. A demonstration of this application is shown in Fig. 3 for the O4 I star, $\zeta$ Pup from Massa et al. (2008). We compare here matches to the FUV PV $\lambda \lambda 1117,1128$ doublet P Cygni profile for (i) the $f$-ratio fixed to the value for the atomic constants $(=2.02)$; and (ii) allowing the $f$-ratio to be a free parameter; a value of 1.84 is adopted for the final least squares fit. This $10 \%$ change in the $f$-ratio undoubtedly improves the match, and is consistent with the evidence for clumping in B supergiants reported in this letter. Finally, we anticipate that the clumping signature contained in the optical depth ratios of doublet lines may also be exploited in other low $v_{\infty}$ settings, such as the fast winds of PN central stars and OB stars in the Magellanic Clouds.

Acknowledgements. D.M. acknowledges support from NASA ADP contract NNH08CD06C to SGT, Inc.

\section{References}

Bouret, J.-C., Lanz, T., \& Hillier, D. J. 2005, A\&A, 438, 301

Cohen, D. J. 2008, in Massive Stars as Cosmic Engines, ed. F. Bresolin, P. A. Crowther, \& J. Puls (Cambridge University Press), Proc. IAU Symp., 250, 17 Fullerton, A. W., Massa, D. L., \& Prinja, R. K. 2006, ApJ, 637, 1025

Ganguly, R., Eracleous, M., Charlton, J. C., \& Chruchill, C. W. 1999, AJ, 117, 2594

Krticka, J., Feldmeier, A., Oskinova, L. M., Kubat, J., \& Hamann, W.-R. 2009, A\&A, 508, 841

Lamers, H. J. G. L. M., Cerruti-Sola, M., \& Perinotto, M. 1987, ApJ, 314, 726

Massa, D., Fullerton, A. W., Sonneborn, G., \& Hutchings, J. B. 2003, ApJ, 586, 996

Massa, D. L., Prinja, R. K., \& Fullerton, A. W. 2008, in Clumping in Hot-Star Winds, ed. W.-R. Hamann, A. Feldmeier, \& L. M. Oskinova, Univ.-Verl., 147 Oskinova, L. M., Hamann, W.-R., \& Feldmeier, A. 2007, A\&A, 476, 1331

Prinja, R. K., Massa, D., \& Fullerton, A. W. 2002, A\&A, 388, 587

Prinja, R. K., Massa, D., \& Searle, S. C. 2005, A\&A, 430, L41

Puls, J., Markova, N., Scuderi, S., et al. 2006, A\&A, 454, 625

Runacres, M. C., \& Blomme, R. 1996, A\&A, 309, 544

Searle, S. C., Prinja, R. K., Massa, D., \& Ryans, R. 2008, A\&A, 481, 777

Sundqvist, J., Puls, J., \& Feldmeier, A. 2010, A\&A, 510, 11

Waldron, W. L., \& Cassinelli, J. P. 2010, ApJ, 711, L30 